\title{
3D Shape Visualization of Curved Needles in Tissue from 2D Ultrasound Images using RANSAC
}

\author{
Michael Waine ${ }^{1}$, Carlos Rossa ${ }^{1}$, Ronald Sloboda ${ }^{2}$, Nawaid Usmani $^{2}$ and Mahdi Tavakoli ${ }^{1}$
}

\begin{abstract}
This paper introduces an automatic method to visualize 3D needle shapes for reliable assessment of needle placement during needle insertion procedures. Based on partial observations of the needle within a small sample of 2D transverse ultrasound images, the 3D shape of the entire needle is reconstructed. An intensity thresholding technique is used to identify points representing possible needle locations within each 2D ultrasound image. Then, a Random Sample and Consensus (RANSAC) algorithm is used to filter out false positives and fit the remaining points to a polynomial model. To test this method, a set of 21 transverse ultrasound images of a brachytherapy needle embedded within a transparent tissue phantom are obtained and used to reconstruct the needle shape. Results are validated using camera images which capture the true needle shape. For this experimental data, obtaining at least three images from an insertion depth of $50 \mathrm{~mm}$ or greater allows the entire needle shape to be calculated with an average error of $0.5 \mathrm{~mm}$ with respect to the measured needle curve obtained from the camera image. Future work and application to robotics is also discussed.
\end{abstract}

\section{INTRODUCTION}

Medical ultrasound is one of the most widely-used imaging modalities for guidance of percutaneous needle insertion procedures such as brachytherapy, biopsy, fluid drainage and anesthesiology. Precise needle tracking within the ultrasound images is crucial for accurate needle positioning. However, there are two main limitations that detract from the effectiveness of ultrasound: 1) The images produced are generally low in quality, and 2) the images often contain false features such as shadows or reverberation artifacts. Additionally, aspects such as needle deflection and tissue deformation cause the needle and desired target location to shift, creating additional challenges. As a result, significant research is directed towards the development of automated methods for locating the needle in ultrasound images. For the purposes of context, our paper will focus discussion towards prostate brachytherapy, but the techniques described are general and can be applied to all forms of ultrasound-guided needle insertion, including both maual and robotically-assisted procedures.

This work was supported by the Natural Sciences and Engineering Research Council (NSERC) of Canada under grant CHRP 446520, the Canadian Institutes of Health Research (CIHR) under grant CPG 127768, and by the Alberta Innovates - Health Solutions (AIHS) under grant CRIO 201201232.

${ }^{1}$ Michael Waine, Carlos Rossa and Mahdi Tavakoli (Corresponding Author) are with the Department of Electrical and Computer Engineering, University of Alberta, AB, Canada T6G 2V4. E-mail: mwaine@ualberta.ca; rossa@ualberta.ca; mahdi.tavakoli@ualberta.ca

${ }^{2}$ Ronald Sloboda and Nawaid Usmani are with the Cross Cancer Institute and the Department of Oncology, University of Alberta, Edmonton, AB, Canada T6G 1Z2. E-mail: \{ron.sloboda, nawaid.usmani\}@albertahealthservices.ca.
A large focus of research has been on the development of needle detection algorithms using 3D volumetric ultrasound images. For instance, Wei et al. [1] developed a needle segmentation algorithm for 3D ultrasound-guided roboticallyassisted brachytherapy. Novotny et al. [2] and Neshat et al. [3] worked on real-time instrument and needle tracking techniques based on the Radon transform. Ding et al. [4] developed a segmentation algorithm designed to locate the needle as well as the seeds implanted during brachytherapy procedures. Uhercik et al. [5] developed a method to detect the position and orientation of long, thin surgical tools using Random Sample and Consensus (RANSAC), which was based on work by Barva et al. [6]. As an extension of [5], Zhao et al. [7], [8] applied a Kalman filter technique and a speckle tracking method to their RANSAC algorithm for tracking micro-tools.

The 3D ultrasound methods mentioned in [1]-[8] are useful for needle segmentation since the entire set of position and orientation parameters can be obtained from a single volume. However, while a few state-of-the-art machines implement special 2D transducer arrays that can allow for real-time 3D imaging, these devices are very costly, and most hospitals will not have access to this type of equipment. It is more common to use ultrasound probes containing 1D transducer arrays to obtain a series of 2D images, and derive the $3 \mathrm{D}$ volume based on their relative positions [9]. With this method, typically less than 1-2 volumes per second can be calculated depending on the sweep speed of the ultrasound probe. As well, only a small segment of the needle can be visualized at once; multiple volumes, each taking several seconds to acquire, are needed in order to visualize the entire needle.

Methods for 2D ultrasound needle segmentation are convenient since the technology is typically cheaper, easier to use, and faster than 3D ultrasound. Research in this field includes work performed by Okazawa et al. [10], who implemented a Hough-transform based detection scheme. Mathiassen et al. [11] developed a real-time algorithm for the estimation of the needle tip. Kaya et al. [12] used Gabor filtering and the utilization of RANSAC to improve needle segmentation. Neubach et al. [13] developed a flexible needle insertion robot guided by $2 \mathrm{D}$ ultrasound images.

Methods [10]-[13] make use of images containing the longitudinal axis of the needle. These images are referred to as "longitudinal" images in our paper, or alternatively, "sagittal" images in the context of prostate brachytherapy in reference to the anatomical planes of the human body. Images of this type are located in the $(y, z)$ plane of Fig. 1. 


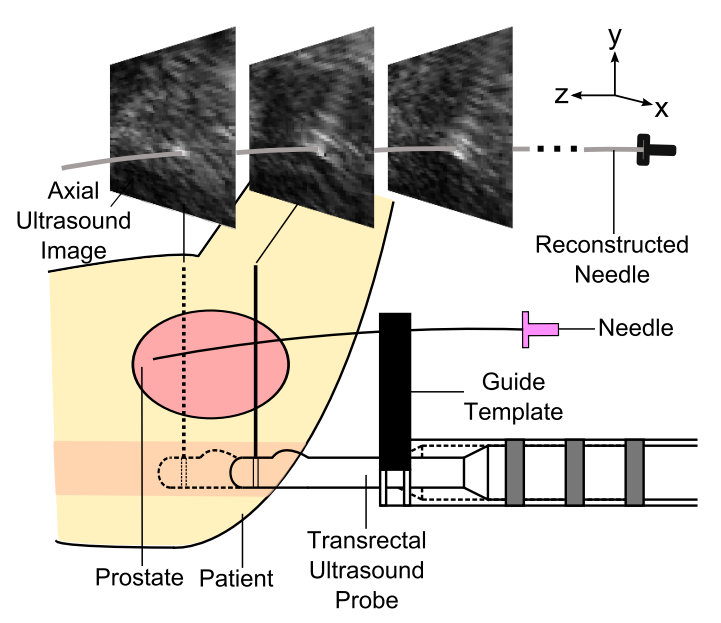

Fig. 1. Depiction of ultrasound-guided prostate brachytherapy. Multiple transverse slices can be obtained by inserting the probe further into the patient. In this paper, the objective is to reconstruct the needle shape based on the needle observations within each transverse slice.

Images perpendicular to the needle's longitudinal axis are named "transverse" images in our paper, or alternatively, "axial" images in prostate brachytherapy. These images are located in the $(x, y)$ plane of Fig. 1. In our paper, the terms longitudinal and transverse will be given preference.

Longitudinal plane imaging is effective when the imaging plane is precisely known; however, aligning the ultrasound probe with the plane of the needle is not trivial, and in many instances tissue motion and needle deflection will cause the needle to diverge from the longitudinal imaging plane. By making use of transverse images, we can avoid the problems of probe alignment found in longitudinal imaging while obtaining a $3 \mathrm{D}$ visualization of the needle shape. The use of transverse images also greatly reduces the amount of data processing required for 3D methods. Finally, in procedures such as prostate brachytherapy, surgeons already monitor several transverse ultrasound slices of the prostate, and so the use of transverse ultrasound images would not only be clinically feasible, but would require very little additional effort by the surgeon.

We propose a new method using $2 \mathrm{D}$ transverse ultrasound images to reconstruct the 3D needle shape. Fig. 1 demonstrates how the ultrasound probe is used in prostate brachytherapy to obtain various transverse views of the needle embedded within the prostate. By varying the position of the probe along the $z$-axis, multiple cross sections of the needle can be obtained. The main premise of this paper is that tracking the movement of the needle cross sections within the plane of each image in conjunction with the relative positions of the images allows for the reconstruction of the entire 3D needle shape. This is implemented using an intensity thresholding technique that locates potential needle locations within each transverse image. A RANSAC algorithm is then used to remove false positives and calculate the needle shape.

The paper is organized as follows. In Section II, the image processing procedure used to identify potential needle locations within the transverse images is summarized. In

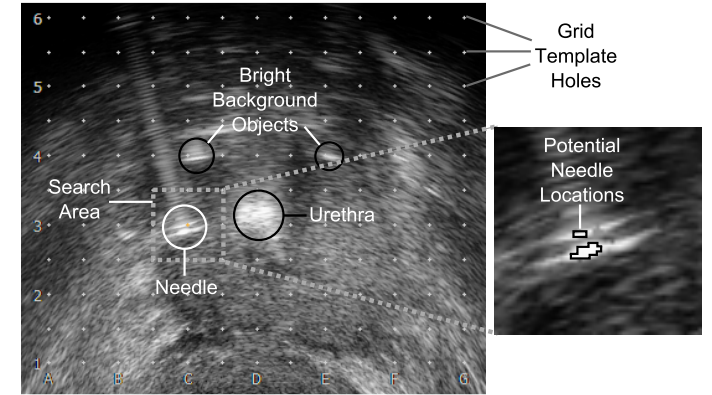

Fig. 2. Example of the needle appearance in 2D transverse prostate brachytherapy images. The needle is circled in white and extraneous objects are circled in black. The image processing result is zoomed-in on the right with the potential needle pixels outlined in black.

Section III, these points are used in a RANSAC curve-fitting algorithm to calculate the curvilinear needle shape. The experimental setup, which acquires multiple transverse ultrasound images along the length of a needle embedded within phantom tissue, is detailed in Section IV. In Section V, results are shown and the accuracy of the proposed method is quantified through comparison with a camera image of the needle shape. Conclusions are drawn in Section VI.

\section{Ultrasound Image Processing}

Each of the 2D transverse ultrasound images must be processed to identify the potential needle locations. In typical transverse ultrasound images, the needle appears throughout each slice as a bright spot. An example is circled in white in Fig. 2. It is often assumed that the needle will be the brightest object in the ultrasound image; however, in brachytherapy for example, a gel is often inserted into the urethra for landmarking purposes, causing the urethra to appear as bright as or brighter than the needle. As well, implanted seeds from previous insertions could create similar bright "background" objects. In Fig. 2, these types of objects are circled in black.

In order to remove the majority of these extraneous background objects from the candidate needle points, the search area for the potential needle pixels is limited to a small window around the needle insertion point, as shown by the gray box in Fig. 2. The needle insertion point can be easily located by aligning the guide template grid with the ultrasound image, demonstrated by the numbered array of gray dots shown in Fig. 2.

After localizing the search area, the MATLAB function imadjust is used to apply an intensity transformation to the cropped image as described in (1).

$$
T(r)=r_{\text {min }}+\left(r_{\text {max }}-r_{\text {min }}\right)\left(\frac{r-r_{\text {low }}}{r_{\text {high }}-r_{\text {low }}}\right)^{\gamma}
$$

where $r, r_{\min }, r_{\max }, r_{\text {low }}$, and $r_{\text {high }}$ are normalized values within the range $[0,1]$. The value $r$ represents the individual pixel intensities within each image, $\left[r_{\min }, r_{\max }\right]$ specifies the minimum and maximum intensities of the desired grayscale spectrum and $\left[r_{l o w}, r_{h i g h}\right]$ specifies the thresholds for saturation at the minimum and maximum grayscale intensities respectively. The desired spectrum is chosen to span $[0,1]$, 


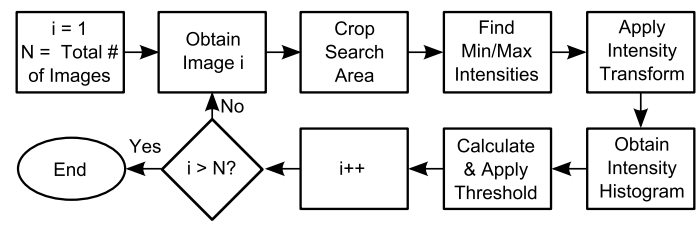

Fig. 3. Flowchart summary of the image processing procedure.

where 0 represents the color black and 1 represents the color white. The saturation thresholds are selected as the minimum and maximum intensity values in the cropped image. The value of $\gamma$ specifies the shape of the mapping curve. By setting $\gamma>1$, the function compresses the gray scale values of the low-intensity pixels, expands those of the high-intensity pixels, and distributes the pixel intensities throughout the entire grayscale spectrum. This allows for improved contrast between the needle and tissue.

Next a histogram of the pixel intensities is obtained, which is used to determine an intensity threshold $\alpha$ such that

$$
\alpha=\min _{r_{n}}\left\{r_{n} \geq \beta \cap n \geq \delta\right\}
$$

where $r_{n}$ is the $n^{\text {th }}$ brightest pixel in the grayscale range, $\beta$ defines the absolute minimum threshold level for $\alpha$, and $\delta$ specifies the number of desired pixels selected for each image. After identifying $\alpha$, the corresponding pixels with intensities greater than $\alpha$ are found. These pixels represent candidate points for the needle location within the transverse image. This image processing procedure is performed for each transverse slice obtained throughout the needle shape. An example of potential needle pixels identified using the above procedure is outlined in black in the zoomed-in portion of Fig. 2 and a summary of the image processing procedure is shown in Fig. 3.

Several pixels are identified after the aforementioned thresholding process, only some of which correspond to the actual needle. Bright background objects can occasionally appear within the search area, and speckle noise often contaminates clinical ultrasound images; these features may make their way through the image processing steps and can be identified as potential needle locations. A method such as least squares curve fitting treats all data points equally, leading to poor results when these types of false positives are present. Therefore, a method is required to identify and exclude outliers before obtaining the needle shape. RANSAC, as described in the next section, can exclude false positives and calculate the needle shape at the same time, making it very suitable for our problem.

\section{RANDom SAMPLE AND CONSENSUS (RANSAC) ALGORITHM}

RANSAC is a robust model-fitting algorithm proficient at handling data sets containing a significant proportion of outliers [14]. The RANSAC Toolbox developed in [15] is used to implement the RANSAC procedure, which is composed of four main steps, namely 1) estimate model

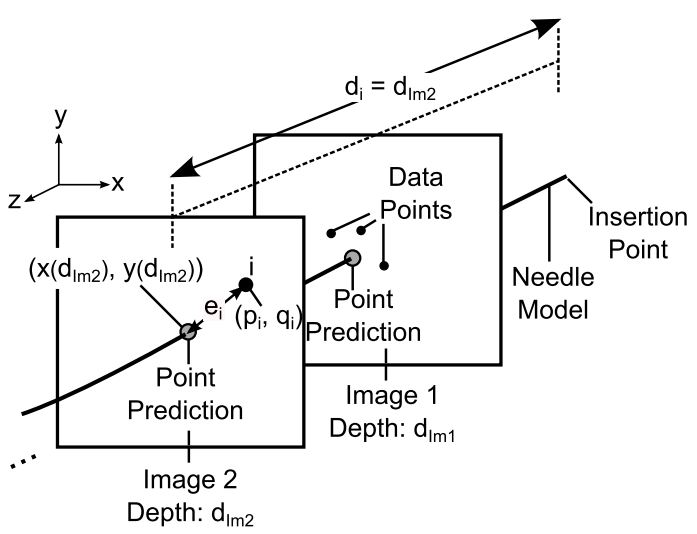

Fig. 4. Diagram showing the calculation of $e_{i}$ for the $i^{\text {th }}$ point in the data set $S$ obtained after image processing.

parameters, 2) develop consensus set, 3) determine the best consensus set, and 4) optimize the results.

1. Estimate Model Parameters: Given a data set $S$ of $N$ points and a model $M$ that requires $k<N$ points for complete parameterization, a random selection of $k$ points is used to identify the model parameters [14]. In our case, $S$ refers to the combined set of $N$ pixel center points identified within all of the transverse images analyzed during the image processing procedure described in Section II, and the needle model $M$ is characterized by the second order polynomial function defined by

$$
\begin{gathered}
x(d)=a_{2} d^{2}+a_{1} d+a_{0} \\
y(d)=b_{2} d^{2}+b_{1} d+b_{0} \\
z(d)=d
\end{gathered}
$$

where $d$ represents the relative needle depth of insertion into tissue with respect to the needle's insertion location and the values $a_{2}, a_{1}, a_{0}$ and $b_{2}, b_{1}, b_{0}$ represent the parameters to be identified. The values $x$ and $y$ refer to the needle's spatial position within the transverse images and $z$ specifies the depth of each image. A second order polynomial is chosen because higher orders result in rippling effects uncharacteristic of the actual needle deflection. In our case, $k=3$ for complete parameterization.

2. Develop Consensus Set: The $i^{t h}$ point in $S$ is defined by the values $\left(p_{i}, q_{i}, d_{i}\right)$ which correspond to the 3D coordinates of the point, as shown in Fig. 4 . The pair $\left(p_{i}, q_{i}\right)$ refers to the spatial location of point $i$ within the transverse image located at depth $d_{i}$. Each point in $S$ is categorized as either an inlier or an outlier based on the magnitude of the error $e_{i}$ between the $i^{\text {th }}$ point and the model we obtained in the previous step using the randomly selected $\mathrm{d}+$ ata points. The value $e_{i}$ can be calculated using (6).

$$
e_{i}\left(d_{i}\right)=\sqrt{\left(x\left(d_{i}\right)-p_{i}\right)^{2}+\left(y\left(d_{i}\right)-q_{i}\right)^{2}}
$$

where $\left(x\left(d_{i}\right), y\left(d_{i}\right)\right)$ represents the model estimation of the needle location at the depth $d_{i}$. An example of $e_{i}$ is demonstrated in Fig. 4, where point $i$ is located in Image 
2. The "Point Prediction" shown in the diagram represents the estimated needle location within each transverse image. In this case, since Image 2 is located at a depth of $d_{\operatorname{Im} 2}$, the Point Prediction for Image 2 is $\left(x\left(d_{I m 2}\right), y\left(d_{I m 2}\right)\right)$. Let us define

$$
\text { inliers }=\left\{\left(p_{i}, q_{i}, d_{i}\right) \mid e_{i}<\varepsilon\right\}
$$

where $\varepsilon$ specifies the inlier tolerance. Points in $S$ that lie further than a distance $\varepsilon$ from their respective Point Predictions are categorized as outliers. The remaining set of inliers is known as the consensus set.

3. Determine Best Consensus Set: Steps 1 and 2 are performed a minimum of $T$ times, and a cost function is evaluated for each consensus set. The M-Estimator Sample Consensus (MSAC) technique developed by Torr et al. [16] is used to obtain the optimal consensus set. This technique is based on the minimization of the cost function $C$ over all points within $S$. The cost function is shown in (8).

$$
C=\sum_{i=1}^{N} f\left(e_{i}\right)
$$

where:

$$
f\left(e_{i}\right)= \begin{cases}e_{i}, & e_{i}<\varepsilon \\ \varepsilon, & e_{i} \geq \varepsilon\end{cases}
$$

Equation (8) is used to rank the consensus sets, where their rankings are inversely proportional to their respective cost function magnitudes. Once the change in the cost function for the highest ranked consensus set becomes smaller than a user-specified tolerance level, the process terminates.

At this point, all false positives are removed from each transverse image slice with respect to the curve obtained from the highest ranked consensus set and a preliminary curve fit describing the needle shape is identified. To summarize the procedure thus far, in Step 1, three randomlyselected points from the data set are used to fit the needle shape model. In Step 2, each point from the entire data set is examined and is categorized as either an inlier or an outlier based on how well it satisfies the model identified in the previous step. In Step 3, the quality of this model is evaluated using the MSAC technique. Steps 1-3 are performed until a model with sufficient quality has been determined.

4. Optimize the Results: The model identified using the RANSAC procedure necessarily passes through at least 3 points within the set of inliers as a result of Step 1. At the end of the process the second order polynomial curve fit is optimized by applying the least-squares method to the entire set of inliers obtained in Step 3 as well as the needle's insertion location. The resulting output is a $3 \mathrm{D}$ approximation of the needle shape.

\section{EXPERIMENTAL SETUP}

A demonstration of the experimental setup used to validate the proposed method is shown in Fig. 5. An 18-gauge, beveltipped brachytherapy needle (Worldwide Medical Technologies) is manually inserted through a stabilizing guide template to a depth of $110 \mathrm{~mm}$ within a transparent tissue

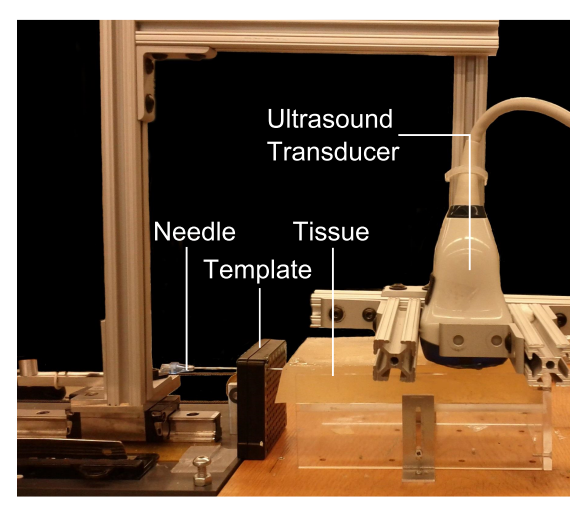

(a) Experimental setup

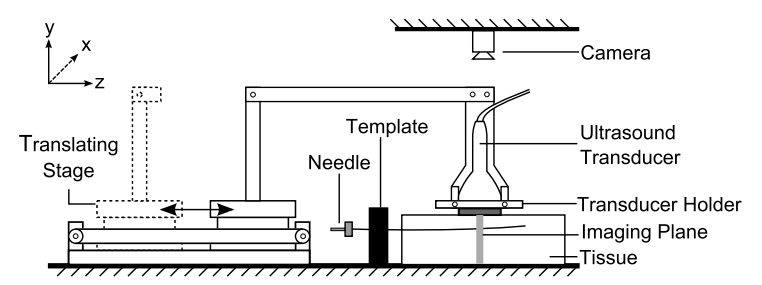

(b) Depiction of the setup mechanics

Fig. 5. A photograph of the setup is shown in (a) and the setup mechanics are depicted in (b). The ultrasound transducer is attached to a translating platform, which is manually adjusted to obtain transverse slices of the embedded needle every $5 \mathrm{~mm}$.

phantom created using a plastisol base (M-F Manufacturing Company). A mechanical holder is used to secure a 4DL145/38 linear ultrasound transducer (Analogic Ultrasound) to a translating stage. The stage is manually positioned to allow for the collection of transverse ultrasound images of the needle every $5 \mathrm{~mm}$ along the $z$-axis, starting from a depth of $5 \mathrm{~mm}$. A total of 21 transverse images are collected using a SonixTouch Ultrasound System (Analogic Ultrasound). As well, a Logitech C270 HD camera mounted above the setup is used to obtain a view of the $(x, z)$ plane of the needle. The needle bevel is positioned such that the out-of-plane needle deflection is negligible; therefore, only the $(x, z)$ plane is required for needle shape comparison. Based on the ultrasound results, the out-of-plane deflection was less than $0.45 \mathrm{~mm}$, which is minor compared to the $11.5 \mathrm{~mm}$ of deflection observed in the $(x, z)$ plane. Needle segmentation from the camera image is performed using morphological image processing, and is used for verification of the RANSAC algorithm results.

The ultrasound images obtained from the experimental system are much clearer than typical medical ultrasound images. To compensate for this, Gaussian noise followed by speckle noise is added to the original images using the function imnoise in MATLAB. A comparison of the results before and after the addition of noise is shown in Fig. 6. The circles highlight the potential needle locations identified using the procedure described in Section II. The addition of noise scatters the potential needle points, similar to results obtained when analyzing clinical images. 


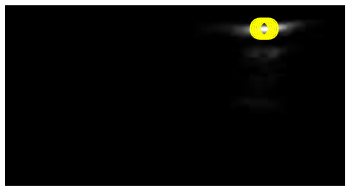

(a) Original image

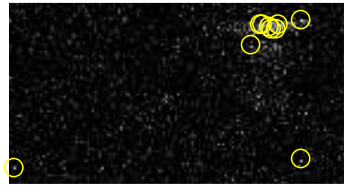

(b) Image after addition of noise
Fig. 6. Example of potential needle points identified using (a) the original image and (b) the images containing artificial noise. The example shown in (b) is closer to the results obtained in clinical ultrasound images.

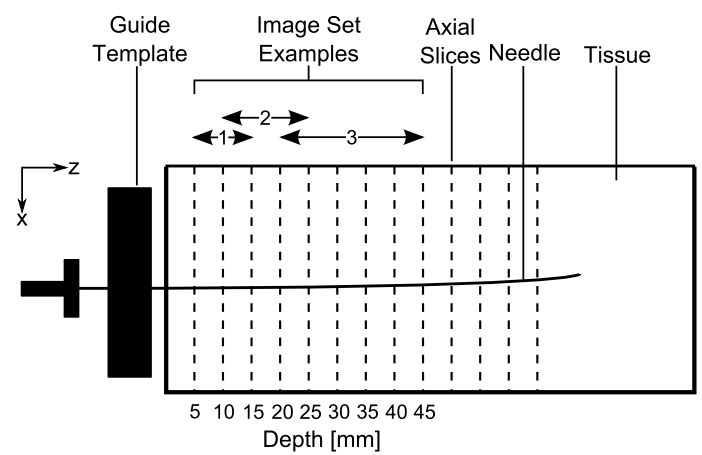

Fig. 7. Example demonstrating how transverse slices were grouped into various image sets. The image sets shown are labelled 1, 2, and 3. Each set starts at a different depth and contains a different portion of the needle.

\section{RESUlts \& Discussion}

The needle model given in (3)-(5) requires at least 3 image slices for full parameterization. The 21 transverse images obtained from the experiment are grouped into sets ranging from 3 successive slices to 21 successive slices, resulting in a total of 190 different image sets. Examples of several different image sets are shown in Fig. 7. The dotted lines represent transverse images taken at various depths along the needle. Example 1 in Fig. 7 contains 3 slices ranging from a depth of $5 \mathrm{~mm}$ to $15 \mathrm{~mm}$, Example 2 contains 4 slices ranging from a depth of $10 \mathrm{~mm}$ to $25 \mathrm{~mm}$, and Example 3 contains 6 slices ranging from $20 \mathrm{~mm}$ to $45 \mathrm{~mm}$.

The RANSAC algorithm is performed on each image set 20 times. The area between the estimated needle shape obtained using the proposed method and the measured needle shape obtained from the camera image is calculated for each iteration. The mean area error is obtained for each image set, and the value is divided by the total insertion depth to obtain the average error between the estimated results and the measured results. A contour plot displaying the results for the 190 image sets is shown in Fig. 8. As well, the maximum error between the predicted needle shape and the measured shape is calculated and the averages are shown in the contour plots of Fig. 9. The $y$-axis represents the difference between the minimum and maximum depths of the image slices considered. This difference is referred to as the "depth differential". Two successive transverse images represents a depth differential of $5 \mathrm{~mm}$, since the images are spaced $5 \mathrm{~mm}$ apart. A set of 3 successive transverse images represents a depth differential of $10 \mathrm{~mm}$ and so on. The $x$ axis shows the depth of the initial slice for each image set.

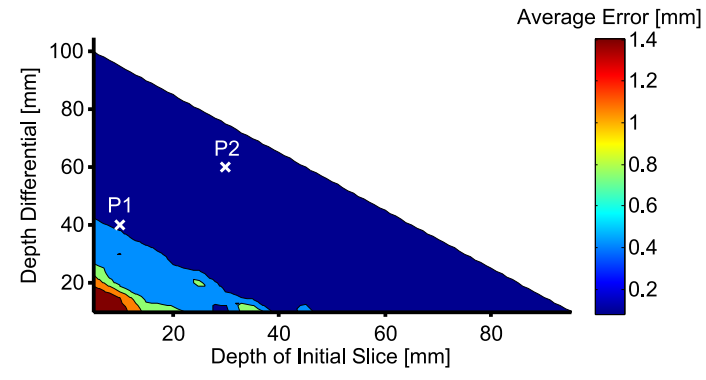

(a) Original images

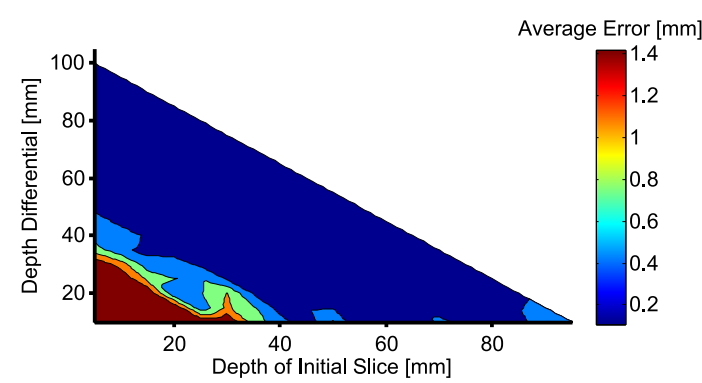

(b) Noisy images

Fig. 8. Contour plots comparing the average error obtained for the (a) original images and (b) noisy images. $P 1$ represents the image set containing a $40 \mathrm{~mm}$ segment of the needle beginning at a depth of $10 \mathrm{~mm} . P 2$ represents a $60 \mathrm{~mm}$ segment starting at a depth of $30 \mathrm{~mm}$.

Therefore, $P 1$ in Fig. 8 represents the image set containing a $40 \mathrm{~mm}$ segment of the needle (or 9 transverse slices) beginning from a depth of $10 \mathrm{~mm} . P 2$ represents a $60 \mathrm{~mm}$ segment starting from a depth of $30 \mathrm{~mm}$. The computations are performed on a PC with a $2.6 \mathrm{GHz}$, AMD Phenom II $x 4$ 910 processor, $4 \mathrm{~GB}$ of RAM, and running 32 bit versions of Windows 7 and MATLAB. Average computation times are $17 \mathrm{~ms}$ to process and locate the needle point candidates in each image, and $107.4 \mathrm{~ms}$ to calculate the needle shape using RANSAC and optimize the solution. The needle shape can be obtained in real-time as soon as the transverse ultrasound images have been collected.

Based on Fig. 8, to obtain an average error of less than 0.5 $\mathrm{mm}$, there exists an inverse relationship between the required depth differential and the starting depth. For example, based on the noisy images, a starting depth of $5 \mathrm{~mm}$ requires a depth differential of $40 \mathrm{~mm}$, whereas a starting depth of $50 \mathrm{~mm}$ only requires a depth differential of $10 \mathrm{~mm}$. A similar trend is observed in Fig. 9, which shows the maximum error between the predicted and measured needle curves. To obtain a maximum error of less than $2 \mathrm{~mm}$, one can image a $50 \mathrm{~mm}$ segment of the needle anywhere along its length, or use an image set containing a $10 \mathrm{~mm}$ depth differential from a starting depth of $50 \mathrm{~mm}$ or greater. Additionally, the results in Figures 8 and 9 are noticeably similar between the normal and noisy images, especially as the depth differential increases, demonstrating the ability of the algorithm to process noise similar to the levels observed in medical ultrasound images.

There are minor inconsistencies for small depth differentials within the noisy images, which can be observed in 


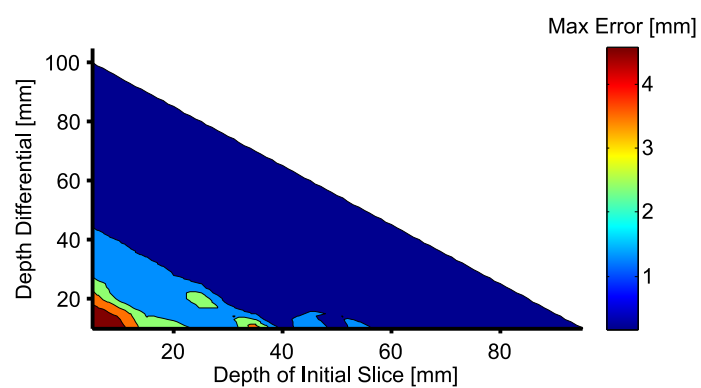

(a) Original images

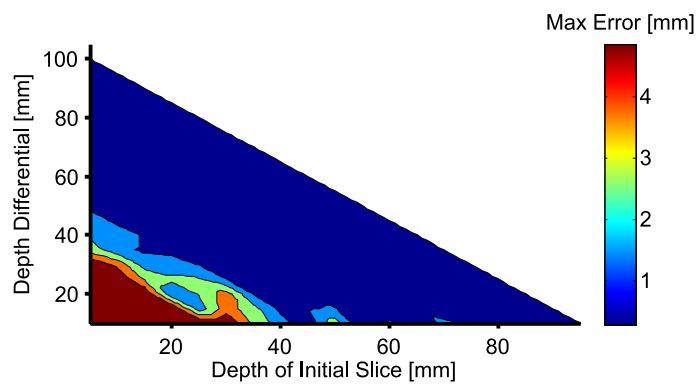

(b) Noisy images

Fig. 9. Contour plots comparing the maximum errors obtained for the (a) original images and (b) noisy images.

the contour formed near the depth of $90 \mathrm{~mm}$ and the depth differential of $10 \mathrm{~mm}$ in panel (b) of Fig. 8. This is likely due to the high degree of extrapolation required when using such a small sample of images.

\section{CONCLUSIONS}

We have presented a method for characterizing the needle shape during needle insertion procedures using $2 \mathrm{D}$ transverse ultrasound images of the embedded needle. An intensity thresholding procedure is used to identify potential needle locations within each transverse slice. Based on the identified points, a RANSAC algorithm is used to remove outliers and statistically parameterize the needle's curvilinear shape.

Experimental results demonstrated that for the collected 2D ultrasound images of the phantom tissue containing the embedded needle, images obtained at starting depths of $50 \mathrm{~mm}$ or greater required only 3 transverse images to obtain a maximum error of less than $2 \mathrm{~mm}$, which is the minimum number of images required for the model parameterization procedure. This type of technology holds great potential for use with robot-guided needle insertion systems. By obtaining the initial needle trajectory across a small sample of transverse slices, the future deflection shape can be predicted and used to align a longitudinal imaging probe for imaging feedback control. The method can also be used for rapid assessment of needle placement in manual prostate brachytherapy. For example, the needle shape within the prostate can be used to assist with the automatic prediction of the final seed placement. The problem of seed placement is highly complex, and requires consideration of additional factors such as seed migration and tissue relaxation following needle retraction, but the proposed method is a necessary first step toward the development of such an automated system.

Although human tissue is inhomogenous, the proposed algorithm is still applicable since needle deflection is only observed, not predicted. However, in future applications such as needle trajectory prediction, additional ultrasound images may be required to improve the accuracy of the predictions. Additional work will focus on assessment of the algorithm using transverse ultrasound images obtained during prostate brachytherapy procedures. As well, the proposed algorithm will be modified to compute the real-time trajectory of the needle, which will be incorporated into an image-guided control system for robotic needle steering applications.

\section{REFERENCES}

[1] Z. Wei, L. Gardi, D. Downey, and A. Fenster, "Oblique needle segmentation for $3 \mathrm{~d}$ trus-guided robot-aided transperineal prostate brachytherapy," in Biomedical Imaging: Nano to Macro, 2004. IEEE Int. Symp., vol. 1, April 2004, pp. 960-963.

[2] P. M. Novotny, J. A. Stoll, N. V. Vasilyev, P. J. Del Nido, P. E. Dupont, T. E. Zickler, and R. D. Howe, "Gpu based real-time instrument tracking with three-dimensional ultrasound," Medical image analysis, vol. 11, no. 5, pp. 458-464, 2007.

[3] H. R. S. Neshat and R. V. Patel, "Real-time parametric curved needle segmentation in 3d ultrasound images," in Biomedical Robotics and Biomechatronics 2008, 2nd IEEE RAS \& EMBS Int. Conf., 2008, pp. 670-675.

[4] M. Ding, Z. Wei, L. Gardi, D. B. Downey, and A. Fenster, "Needle and seed segmentation in intra-operative $3 \mathrm{~d}$ ultrasound-guided prostate brachytherapy," Ultrasonics, vol. 44, pp. e331-e336, 2006.

[5] M. Uhercik, J. Kybic, H. Liebgott, and C. Cachard, "Model fitting using ransac for surgical tool localization in 3-d ultrasound images," Biomedical Engineering, IEEE Transactions on, vol. 57, no. 8, pp. 1907-1916, 2010.

[6] M. Barva, J. Kybic, J.-M. Mari, C. Cachard, and V. Hlavác, "Automatic localization of curvilinear object in $3 \mathrm{~d}$ ultrasound images," in Medical Imaging, 2005, pp. 455-462.

[7] Y. Zhao, H. Liebgott, and C. Cachard, "Tracking micro tool in a dynamic 3d ultrasound situation using kalman filter and ransac algorithm," in Biomedical Imaging (ISBI), 2012 9th IEEE Int. Symp., 2012, pp. 1076-1079.

[8] Y. Zhao, H. Liebgott, C. Cachard et al., "Tracking biopsy needle using kalman filter and ransac algorithm with $3 \mathrm{~d}$ ultrasound," Acoustics 2012, pp. 231-236, 2012.

[9] A. Fenster. (2008, Dec.) 3-dimensional ultrasound imaging. Imaging Economics. [Online]. Available: http://www.imagingeconomics.com/ 2004/12/3-dimensional-ultrasound-imaging/

[10] S. H. Okazawa, R. Ebrahimi, J. Chuang, R. N. Rohling, and S. E. Salcudean, "Methods for segmenting curved needles in ultrasound images," Medical image analysis, vol. 10, no. 3, pp. 330-342, 2006.

[11] K. Mathiassen, D. Dall'Alba, R. Muradore, P. Fiorini, and O. J. Elle, "Real-time biopsy needle tip estimation in $2 \mathrm{~d}$ ultrasound images," in Robotics and Automation (ICRA), 2013 IEEE Int. Conf., 2013, pp. 4363-4369.

[12] M. Kaya and O. Bebek, "Needle localization using gabor filtering in 2d ultrasound images," in Robotics \& Automation (ICRA), 2014 IEEE International Conf., 2014, pp. 4881-4886.

[13] Z. Neubach and M. Shoham, "Ultrasound-guided robot for flexible needle steering," Biomedical Engineering, IEEE Transactions on, vol. 57, no. 4, pp. 799-805, 2010.

[14] M. A. Fischler and R. C. Bolles, "Random sample consensus: a paradigm for model fitting with applications to image analysis and automated cartography," Communications of the ACM, vol. 24, no. 6 , pp. 381-395, 1981.

[15] M. Zuliani. (2008, Nov.) Ransac toolbox for matlab. [Online]. Available: http://vision.ece.ucsb.edu/ zuliani/Research/ RANSAC/RANSAC.shtml

[16] P. H. Torr and A. Zisserman, "Mlesac: A new robust estimator with application to estimating image geometry," Computer Vision and Image Understanding, vol. 78, no. 1, pp. 138-156, 2000. 